\title{
Characterizing a Novel Food Waste Recovery Process Using an Electrostatic Separator
}

\author{
Koonchun Lai*, Sooking Lim, Pehchiong Teh, Kimho Yeap \\ Universiti Tunku Abdul Rahman, Perak, Malaysia
}

Received: 20 January 2016

Accepted: 24 April 2016

\begin{abstract}
Organic food waste compounds in landfills can heavily pollute the soil, aquifer, and air, mainly due to anaerobic transformation. This paper presents the outcomes of research on the waste separation process and recovery of food waste (FW) using an electrostatic separator. The separation process sorts the charged bodies from the uncharged under an intensive electric field. We studied influences of various parameters such as applied voltage, rotation speed, and electrode angle on the separation process. Results revealed that separation efficiency relies on a uniform electric field and separator configuration. To our best knowledge, this is the first characterization report for food waste recovery using an electrostatic separator.
\end{abstract}

Keywords: waste recovery, separation, electric field, food waste management

\section{Introduction}

Waste management is an inevitable and critical global challenge facing most countries in the world [1-2]. A high amount of organic matter - particularly food waste - is the principal component of the waste stream [3]. Wasting food has become a global problem in recent decades [4], and food waste residues are in general turned into landfills. However, the decomposition of organic substances leads to the greenhouse phenomenon [5]. Lately, researchers have placed high emphasis in food waste since the high organic content in food waste has the potential to be turned into valuable products such as bioethanol and biobutanol [6-7]. Bioethanol is one of the most promising alternative energy sources that can help decrease global dependence on fossil fuels [8]. It can be produced by the fermentation of sugar-rich crops and food wastes. Besides, proper treatment of organic food waste (e.g., anaerobic digestion) enables the harnessing of biogas and production of bioenergy [9].

In the present study we utilized an electrostatic separator to segregate food waste and non-food waste from waste mixtures. An electrostatic separator is capable of separating particles based on the conductivities of the constituent components. It is widely used to sort out particles with high conductivities from those with relatively low conductivities (e.g., to recover conductors from nonconducting mixtures). It serves as an environmentally friendly way for recycling and reusing the resources without negatively impacting the surroundings [10]. At present, an electrostatic separator has been used to separate particles for various applications [11-12]. However, to date there has been a lack of progress made in food waste recovery [13]. This paper investigates the influences of various parameters of the separator on the recovery process of food waste. To our best knowledge, this is the first characterization report for food waste recovery using an electrostatic separator.

*e-mail: laikc@utar.edu.my 


\section{Material and Methods}

The test sample was typically a mixture of food waste particles (40 wt $\%$ fruit peel) and non-food waste particles (10 $\mathrm{wt} \%$ glass and $50 \mathrm{wt} \%$ plastic) prepared in $100 \mathrm{~g}$ samples. The size of each particle averaged $3.0 \mathrm{~mm}$. We employed a roll-type electrostatic separator [14]. The waste mixture eventually dropped into three collection tanks, namely a food waste (FW) tank, a non-food waste (NF) tank, and a middling (M) tank. The mass from each tank was measured by a digital precision balance with a resolution of $0.01 \mathrm{~g}$ after each run. The recovery, $R(\%)$ of food waste, $R_{F W}$ and non-food waste, and $R_{N F}$ were calculated by:

$$
\begin{gathered}
\% R F W, P_{F W}=\frac{m_{F W}}{F W_{0}} \times 100 \% \\
\% R N F, R_{N F}=\frac{m_{N F}}{N F_{0}} \times 100 \%
\end{gathered}
$$

$\ldots$ where $m_{F W}, F W_{0}, m_{N F}$, and $N F_{0}$ are the mass in the FW tank, initial food waste mass in the feeder, mass in the NF tank, and initial non-food waste mass in the feeder, respectively. Similarly, the grades of the food waste tank $\left(G_{F W}\right)$ and non-food waste tank $\left(G_{N F}\right)$ were determined by:

$$
\begin{aligned}
& \% G F W, G_{F W}=\frac{m_{F W}^{\prime}}{m_{F W}} \times 100 \% \\
& \% G N F, G_{N F}=\frac{m_{N F}^{\prime}}{m_{N F}} \times 100 \%
\end{aligned}
$$

... where $m_{F W}^{\prime}$ and $m_{N F}^{\prime}$ are the food waste mass in the FW tank and non-food waste mass in the NF tank, respectively. Both $m_{F W}^{\prime}$ and $m^{\prime}{ }_{N F}$ were determined by manual sorting.

The extent of electrostatic separation caused by applied voltage could be quantified in terms of food waste recovery and middling product yield. High recovery with less middling is desired for an efficient separation process. Recovered food waste (FW) refers to the multiplication product of recovery $(\% \mathrm{RFW})$ and grade $(\% \mathrm{GFW})$ with the initial food waste feed measured in mass. In an attempt to make a comprehensive understanding on the separation process of food waste, this study explores the effect of various operating conditions in terms of the applied voltage, roller rotation speed, and position of the electrodes of the electrostatic separator.

\section{Results and Discussion}

Fig 1 illustrates the effects of applied voltage on food waste recovery and grade (Fig. 1a), recovered food mass and middling (Fig. 1b), and grade and recovered non-food mass (Fig. 1c). It was apparent that the separation and recovery processes were related significantly to the applied voltage. On the other hand, the relationship between the voltage and the recovery at different rotation speeds were depicted in Fig. 2. The separation of food waste at slower rotation speed was found to be more effective. The results were confirmed by the decline in middling mass.

Fig. 3 shows the results for the change of the FW recovery with different electrode angle designs under

a)

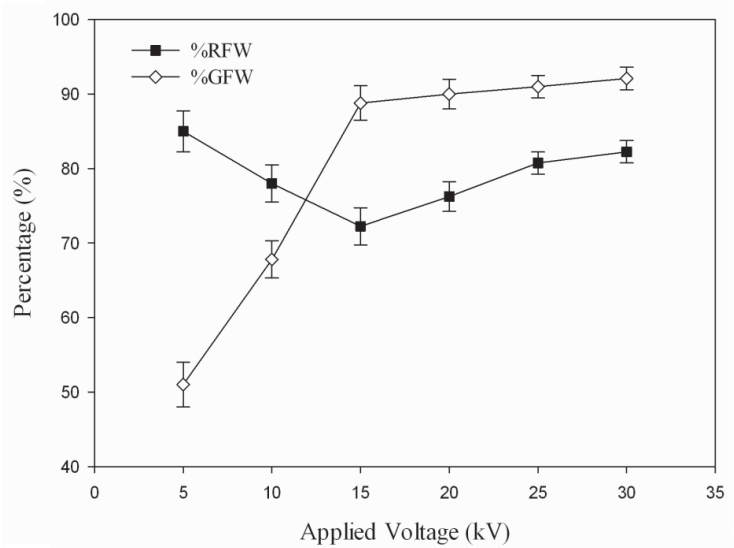

b)

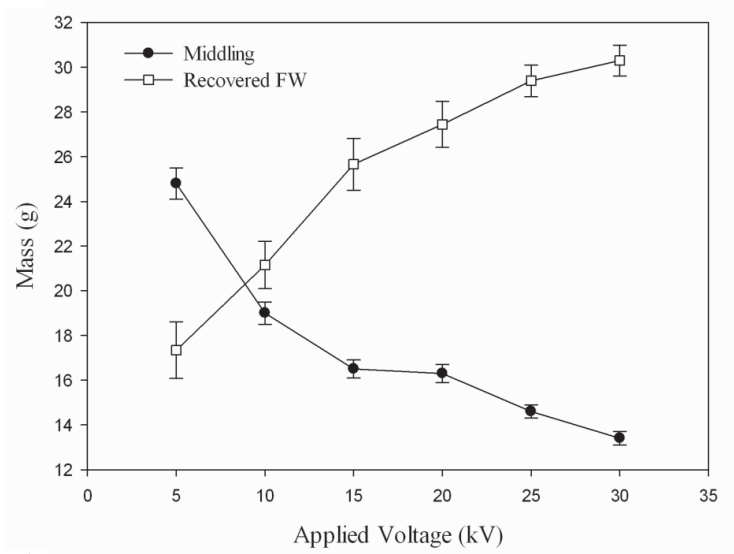

c)

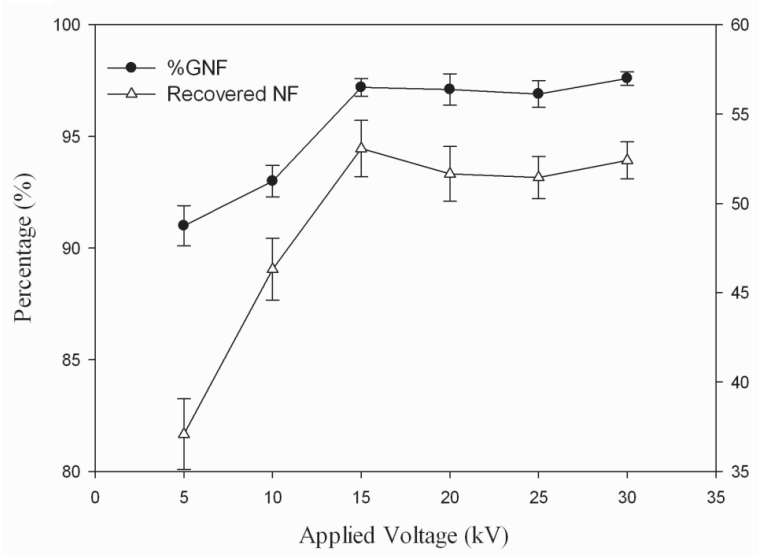

Fig. 1. Effects of applied voltage on a) food waste recovery and grade, b) mass of recovered food waste and middling, and c) grade and recovered mass of non-food waste (rotation speed $=$ 70 rpm; feed content FW:NF = 40:60). 
various applied voltages. A significant separation was noticed for a large angle of 50 degrees, whereas a smaller angle of 25 degrees exhibited marginal improvement of efficiency. The influence of electrode angles on the separation process is also reflected from the middling mass measurement. The grade and mass of samples for FW and NF along with the mass of middling under an applied voltage of $25 \mathrm{kV}$ are shown in Fig. $3 \mathrm{~b}$.

\section{Force Model}

The electrostatic separation process relies on forces act on the particles, allowing them to be sorted out from the mixture. A number of forces exist in the process [15-16]. This includes the pinning force induced by the corona electrode and the mechanical centrifugal force due to rotation. Figure 4 illustrates the forces that act on a particle during the separation process. The four forces were summarised as (i) centrifugal force due to rotation, (ii) lifting force due to attraction by the electrostatic electrode, (iii) gravity force, and (iv) pinning force due to ion generation from the corona electrode on the insulative particles.

a)

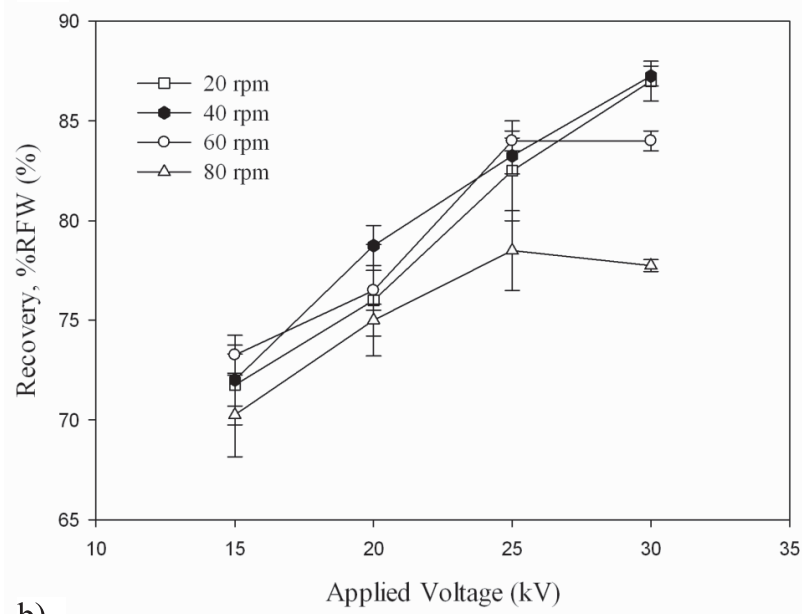

b)

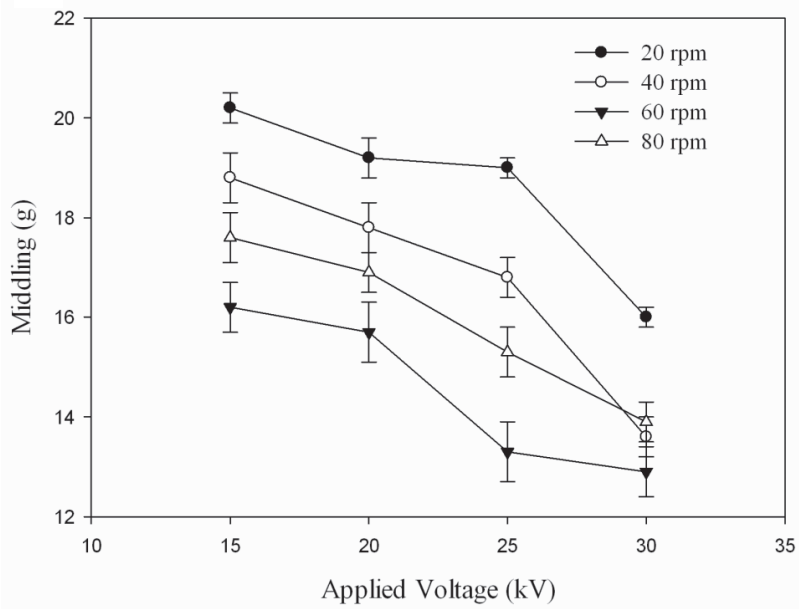

Fig. 2. Effect of roller rotation speed on a) FW recovery and b) middling (feed content $\mathrm{FW}: \mathrm{NF}=40: 60$ ).

\section{Effect of Applied Voltage}

Corona discharge from the corona electrode is generated continuously once the power supply is turned on, and ceased immediately as soon as the power is cut off. A drastic increment of grade $(\% \mathrm{GFW})$ along with a distinct rise of recovery ( $\% \mathrm{RFW})$ was noted at the voltage level of above $15 \mathrm{kV}$ in Fig. 1. For instance, in Fig. 1a, the grade was improved from $51 \%$ to $89 \%$ when the voltage increased from $5 \mathrm{kV}$ to $15 \mathrm{kV}$. The observation suggested that the threshold voltage existed in the electrostatic separation process. It was in good agreement with the studies of [17]. Therefore, threshold voltage (i.e., $15 \mathrm{kV}$ ) should be set for the parametric studies throughout this study. Although high separation efficiency can be observed at $5 \mathrm{kV}$, it in fact resulted in a very low purity and consequently poor food waste recovery, mainly due to the weak electric field.

To better understand the extent of applied voltage on the separation process, the weight of middling products and the recovered food waste were measured. As shown in Fig. 1b, it can be observed that the recovered food waste increases with the increase of applied voltage

a)

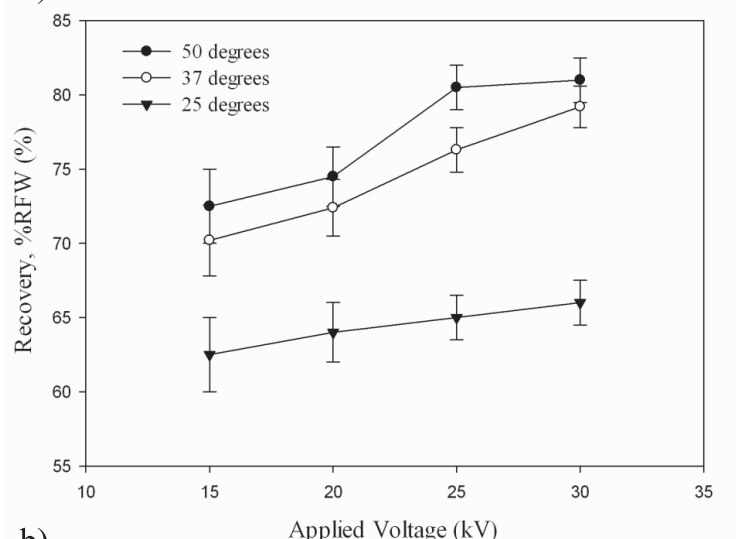

b)

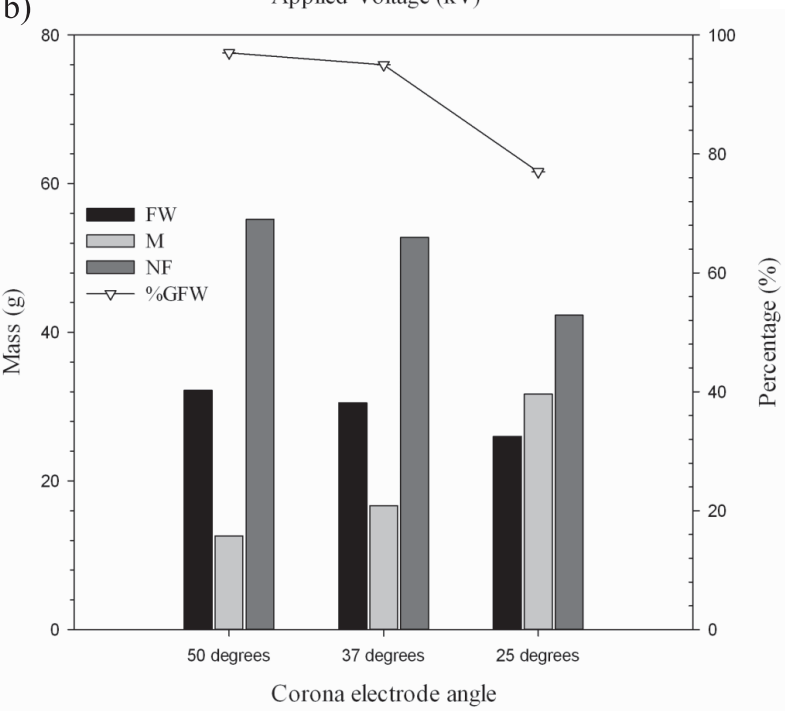

Fig. 3. a) Effect of electrode angle on FW recovery and b) Mass and grade with different electrode angle (rotation speed $=70$ rpm; feed content FW:NF = 40:60). 
and reduction of middling products. The results were similar to the findings of different applications using the electrostatic separator [18]. This can be interpreted as a stronger electric field enhancing the detachment of food waste to the FW tank as well as the separation grade, thus reducing the mass in the middling collection tank. It has been established that the separation process is attributed to electrostatic force, $F_{e}$ :

$$
F_{e}=Q E=K \varepsilon E^{2} \rho_{c}
$$

...where $K$ is a constant, $\varepsilon$ is dielectric constant of particle, $E$ is electric field strength, and $\rho_{c}$ is surface charge density. As expressed by Eq. (1), the electrostatic force $F$ is directly proportional to the square of the electric field. It can be deduced that the higher the electric field, the higher the electrostatic force acted to deviate food particles from a falling trajectory, which leads to better separation efficiency and food recovery [19].

Fig. 1c illustrates the effect of applied voltage on the non-food particles. It showed that the high grade of nonfood particles (more than $90 \%$ in this case) was found inside the NF collection tank with insignificant impurities. However, the recovered mass of NF, which derived from the multiplication product of NF recovery (\%RNF) and grade (\%GNF), exhibited a comparatively low output when the applied voltage was below $15 \mathrm{kV}$. This was due to the fact that the low electric field is insufficient to generate a strong pinning force to move NF particles into the NF tank [20]. Besides, the results verified the previous discussion that the minimal required voltage level should be set to obtain effective separation results. In order to ensure that the particle drops into the non-food collection tank, the following conditions have to be satisfied:

$$
F_{i}+F_{g} \cos \alpha \geq F_{c t}
$$

and

$$
F_{i} \geq F_{c t}+F_{g}, \text { when } \alpha=\pi
$$

\section{Effect of Roller Rotation Speed}

The separation of food waste at slower rotation speed was found to be more effective, as seen in Fig. 2. Fig. 2a shows that at the same applied voltage of $30 \mathrm{kV}$, FW recovery can reach about $87 \%$ at a speed of $20 \mathrm{rpm}$ if compared to $77 \%$ at $80 \mathrm{rpm}$. It appeared that the lowest level of rotation speed can prolong the time of waste particles to stay within the ionising zone. Nevertheless, the slow processing speed is impractical for energy cost optimisation. As such, the speed range of $40 \mathrm{rpm}$ to $80 \mathrm{rpm}$ could be considered for a rapid separation process on one hand, and for maintaining a high recovery of waste on the other. As shown in Fig. 2b, in general the middling mass reduced with faster roller rotation, indicating an enhancement to separation efficiency, which is in contrast to the previous discussion. In fact, centrifugal force $F_{c t}$ relates positively to the rotation speed, calculated as:

$$
F_{c t}=m \omega^{2} R
$$

... where $R$ is the radius of a separator roller, $\omega$ is the angular velocity, and $m$ is the mass of the particle. In other words, a higher rotation speed implies a larger centrifugal force to deviate the food particles to the FW tank. Nevertheless, results in this section exhibited a slower rotation process that can also contribute to effective separation. The separation performance in this case can be regarded as a competition between centrifugal force and ionising time $[12,21]$. There is likely a critical roller rotation speed level, and it should be determined specifically via an optimisation methodology as a future work.

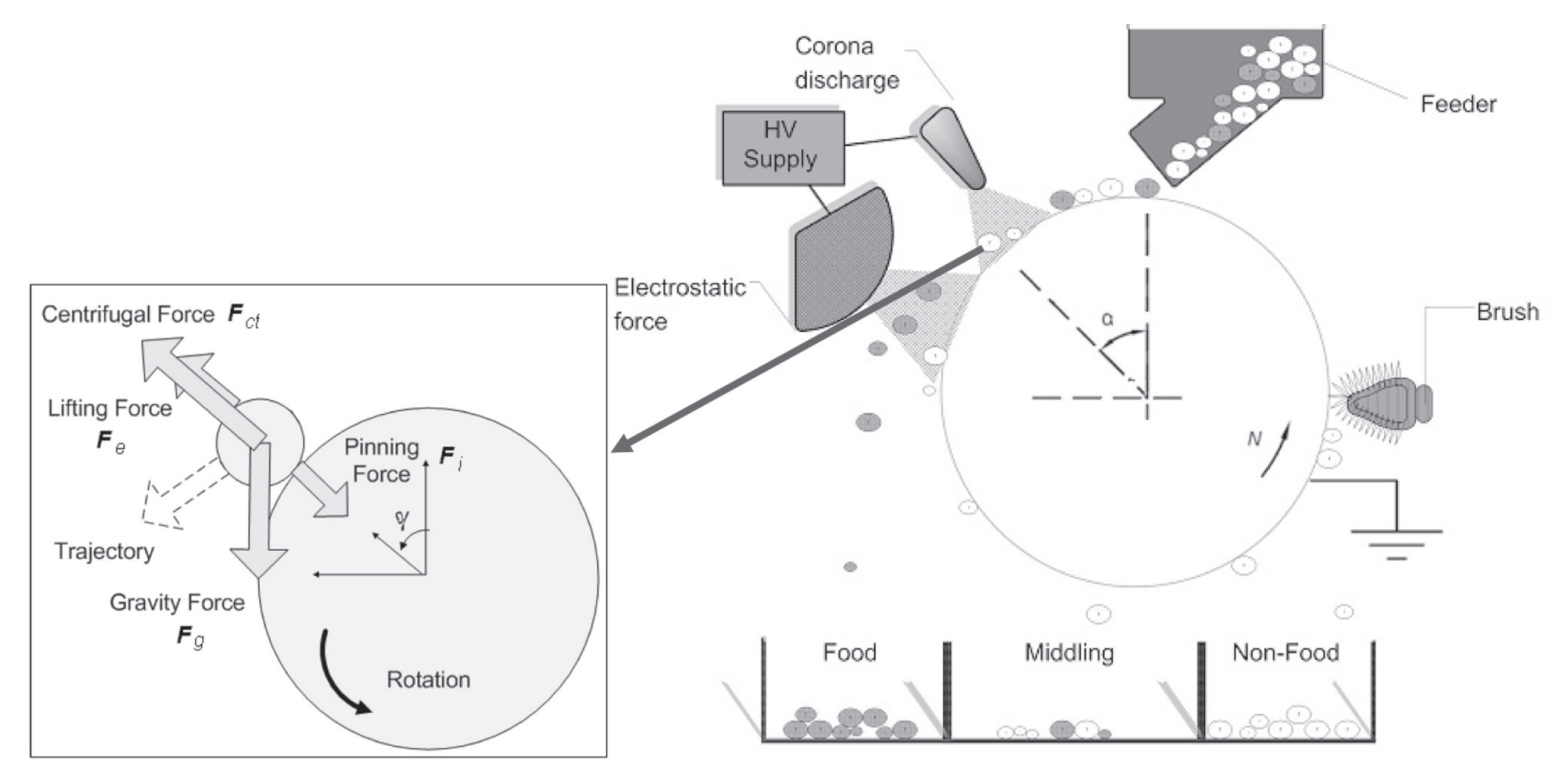

Fig. 4. Separation process and various potential forces acting on particles. 


\section{Effect of Angular Position of Electrodes}

As depicted in Fig. 3, great reduction in middling was accomplished with the electrode angle (denoted as $\alpha$ in Fig. 4) of 50 degrees at the same applied voltage compared to that of 37 degrees and 25 degrees. It appears that the separation efficiency would be enhanced in terms of reduction of middling with larger $\alpha$. Set on a basis that the intensity of space charge between the electrodes increases with a closer distance would yield higher efficiency. However, it is also possible that if the electrodes are located too close, the charge would gather around the two electrodes and cause non-uniform charge distribution [22].

Moreover, it is noticeable in Fig. $3 b$ that the grade and recovery decreased with the decrement of $\alpha$. The reason for the deduction could be due to the reduction in the electric field strength that corresponds to the corona electrode angle [23]. Besides, it could be due to the fact that the corona electrode was placed too close to the feeder output. Based on the experimental observation when the angle of corona electrode was set at 25 degrees, the large coverage of the induced ionising charge interferes with the particles coming out from the feeder. The charge repels the particle undesirably instead of pinning it onto the surface of the roller. In other words, with the small angle of corona electrode, the particle could eventually fall into the NF tank or be classified as middling if it were to fall outside any tank, thereby increasing the mass of middling and affecting the NF collection grade.

\section{Conclusion}

The applied voltage, roller rotation speed, angular position of electrodes, and composition of the mixture were examined on their impacts on the separation process. We can summarise from the parametric studies and theoretical consideration that the most superior effect of electrostatic separation was associated with a test sample under a high voltage level. The separation process can be enhanced by determining the optimum values of rotation speed and electrode gap for creating an intensive charging coverage, while keeping the process time short to save energy. The optimisation model could be established as it would provide a comprehensive analysis for enhancing separation efficiency.

\section{References}

1. PROKIC D., MIHAJLOV A. Contaminated sites. Practice of solid waste management in a developing country (Serbia). Environ. Prot. Eng. 38 (1), 81, 2012.

2. MALLAK S.K., ISHAK M.B., KASIM M.R.M., SAMAH M.A.A. Assessing the effectiveness of waste minimization methods in solid waste reduction at the source by manufacturing firms in Malaysia. Pol. J. Environ. Stud. 24 (5), 2063, 2015.

3. AHSAN A., ALAMGIR M., IMTEAZ M., SHAMS S.,
ROWSHON M.D.K., AZIZ M.G. et al. Municipal solid waste generation, composition and management: Issues and challenges. A case study. Environ. Prot. Eng. 41 (3), 43, 2015.

4. ERIKSSON, M., STRID, I., HANSSON, P.-A. Food waste reduction in supermarket - Net costs and benefits of reduced storage temperature. Resour. Conserv. Recy. 107, 73, 2016.

5. STASZEWSKA E., PAWLOWSKA M. Characteristics of emissions from municipal waste landfills. Environ. Prot. Eng. 37 (4), 119, 2011.

6. LE MAN H., BEHERA S.K., PARK H.S. Optimization of operational parameters for ethanol production from Korean food waste leachate. Int. J. Environ. Sci. Technol. 7, 157, 2010.

7. MOUKAMNERD C., KAWAHARA H., KATAKUR Y. Feasibility study of ethanol production from food Wastes by consolidated continuous solid-state fermentation. J. Sust. Bioenerg. Sys. 3, 143, 2013.

8. MCMILAN J.D. Bioethanol production: status and prospects. Renew. Energ. 10, 295, 1997.

9. KHAIRUDDIN N., MANAF L.A., HASSAN M.A., HALIMOON N., KARIM W.A.W. Biogas harvesting from organic fraction of municipal solid waste as a renewable energy resource in Malaysia: A review. Pol. J. Environ. Stud. 24 (4), 1477, 2015.

10. KIEWIET C., BERGOUGNOU M.A., BROWN J.D. Electrostatic separation of fine particle in vibrates fluidized beds. IEEE T. Ind. Appl. 6, 526, 1978.

11. VEIT H.M., DIEHL T.R., SALAMI A.P., RODRIGUES J.S., BERNARDES A.M., TENORIO J.A.S. Utilization of magnetic and electrostatic separation in the recycling of printed circuit boards scrap. Waste Manage. 25, 67, 2005.

12. XUE M., LI J., XU Z. Environmental friendly crushmagnetic separation technology for recycling metal-plated plastics from end-of-life vehicles. Environ. Sci. Technol. 46, 2661, 2012.

13. LIN C.S.K., PFALTZGRAFF L.A., HERRERO-DAVILA L., MUFOBU E.B., ABDERRAHIM S., CLARK J.H. et al. Food waste as a valuable resource for the production of chemicals, materials and fuels. Current situation and global perspective. Energ. Environ. Sci. 6, 426, 2013.

14. LAI K., LIM S., TEH P. Optimization of electrostatic separation process for maximizing biowaste recovery using Taguchi method and ANOVA. Pol. J. Environ. Stud. 24 (3), 1125, 2015.

15. DAS S., MEDLES K., YOUNES M., DRAGAN C., DASCALESCU, L. Separation of fine granular mixtures in s-plate-type electrostatic separators. IEEE T. Ind. Appl. 43, 1137, 2007.

16. XU Z.M., LI J., LU H.Z., WU, J. Dynamics of conductive and nonconductive particles under high-voltage electrostatic coupling fields. Sci. China Ser E. 52, 2359, 2009.

17. KAWAMOTO H., UMEZU S. Development of electrostatic paper separation and feed mechanism. J. Electrostat. 65, 438, 2007.

18. HOU S., WU J., QIN Y., XU Z. Electrostatic separation for recycling waste printed circuit board: a study on external factor and a robust design for optimization. Environ. Sci. Technol. 44, 5177, 2010.

19. BENDAOUD A., TILMATINE A., MEDLES K., RAHLI M., HUZAU M., DASCALESCU, L. Charaterization of dual corona-electrostatic electrodes for electrostatic processes applications. IEEE T. Ind. Appl. 44, 692, 2008.

20. YOUNES M., TILMATINE A., MEDLES K., BENDAOUD A., SAMUILA A., DASCALESCU L. Numerical modeling 
of insulating particle trajectories in roll-type coronaelectrostatic separators. IEEE T. Dielectr. Electr. Insul. 16, 629, 2009.

21. ELDER J., YAN E., RAISER G. eForce - Newest generation of electrostatic separator for the mineral sands industry. In: Heavy Minerals Conference. South Africa Institute of Mining and Metallurgy, Johannesburg, 2003.

22. BANERJEE S., HOFFMEISTER B.K., SVEJKOSKY
J.J., BADGER C.S. Electrostatic charging of a conducting sphere using another conducting sphere at constant voltage. J. Electrostat. 69, 601, 2011.

23. AMAN F., MORAR R., KOHNLECHNER R., SAMUILA A., DASCALESCU L. High-voltage electrode position: a key factor of electrostatic separation efficiency. IEEE T. Ind. Appl. 40, 905, 2004. 